\title{
On Quasinormal Modes for Scalar Perturbations of Static Spherically Symmetric Black Holes in Nash Embedding Framework
}

\author{
Sergio C. Ulhoa, ${ }^{1}$ Ronni G. G. Amorim, ${ }^{2}$ and Abraão J. S. Capistrano ${ }^{3}$ \\ ${ }^{1}$ Instituto de Física, Universidade de Brasília, 70910-900 Brasília, DF, Brazil \\ ${ }^{2}$ Faculdade Gama, Universidade de Brasília, Setor Leste (Gama), 72444-240 Brasília, DF, Brazil \\ ${ }^{3}$ Casimiro Montenegro Filho Astronomy Center, Itaipu Technological Park, Federal University of Latin-American Integration, \\ P.O. Box 2123, 85867-970 Foz do Iguaçu, PR, Brazil
}

Correspondence should be addressed to Sergio C. Ulhoa; sc.ulhoa@gmail.com

Received 15 October 2016; Revised 21 December 2016; Accepted 15 January 2017; Published 14 February 2017

Academic Editor: Torsten Asselmeyer-Maluga

Copyright (C) 2017 Sergio C. Ulhoa et al. This is an open access article distributed under the Creative Commons Attribution License, which permits unrestricted use, distribution, and reproduction in any medium, provided the original work is properly cited. The publication of this article was funded by SCOAP ${ }^{3}$.

In this paper we investigate scalar perturbations of black holes embedded in a five-dimensional bulk space. The quasinormal frequencies of such black holes are calculated using the third order of Wentzel, Kramers, and Brillouin (WKB) approximation for scalar perturbations. The high overtones of quasinormal modes indicate a resonant-like set of black holes suggesting a serious constraint of embedding models in five dimensions.

\section{Introduction}

The black hole solutions can be perturbed by some matter field; then it leads to oscillations, which can be explained by an analogy with a vibrating string; in such a system the oscillations decrease with time as the string loses energy to the environment [1]. Similarly to the string, where its frequency can be expressed by a complex parameter called quasinormal frequency, the perturbations of black holes evolve with time as an oscillation with complex frequency, in which the real part is responsible for the period of oscillation and the imaginary part is responsible for damping or amplification. Thus proper oscillations of black holes are also called quasinormal modes.

Historically, the theoretical study on perturbations of black holes began with the seminal paper of Regge and Wheeler in 1957 in which they studied the stability of Schwarzschild black hole submitted to small perturbations [2]. In the 70s, the quasinormal modes were used by Vishveshwara in calculations of scattering of gravitational waves by a Schwarzschild black hole [3]. Since then, the study of this issue was directed to several problems. Black holes stability is an example of this importance. The relevance of studying the stability of these astrophysical objects is the possibility of understanding theories in higher dimensions as aspects of brane-world and string theory $[4,5]$ and variants. Since the uniqueness theorem is not applicable to more than four dimensions, stability can be the criterion to select physical solutions [6,7]. Another important application of the quasinormal modes of black holes is in the study of the ADS/CFT correspondence [8], whereby the imaginary part of the quasinormal fundamental frequency describes the thermalization time in a conformal invariant field theory in the border of the anti-de-Sitter space-time. In addition, there are prospects that in the near future more oscillations of astrophysical black holes can be observed using gravitational waves detectors as in LIGO Scientific Collaboration and Virgo Collaboration [9] and be an important tool to constrain higher dimensional models [10, 11]. This fact is relevant in the sense that quasinormal modes carry information about stellar objects, such as mass, charge, and angular momentum.

Considering the relevance of the quasinormal modes, in this work we study scalar perturbation of a black hole 
embedded in a five-dimensional bulk space by calculating its quasinormal frequencies. In this sense, this paper is organized as follows. In Section 2, we present a summary of the Nash embedding theorem to study the induced four-dimensional equations embedded in a five-dimensional bulk space. In Section 3, we present the induced potential in embedded spherically symmetric vacuum solution. In Section 4, we discuss scalar perturbations. In Section 5, we present the quasinormal modes for treated system using $\mathrm{WKB}$ approach and the resulting effective potential. In Section 6, we present our concluding remarks.

\section{Embedded Induced Four-Dimensional Equations}

The proof of the Poincaré conjecture by Perelman [12, 13] suggests a new paradigm for geometry and in particular for Einstein's gravitation and cosmology, namely, the possibility that we can deform space-time in arbitrary directions, changing its shape. It has its origins from a solution for the Riemann curvature ambiguity was conjectured by L. Schlaefli in 1871, proposing that the Riemann manifolds should be embedded in a larger bulk space, such that its Riemann curvature $\mathbb{R}$ would act as a curvature reference for all embedded manifolds, just like the flat Euclidean space $\mathbb{R}^{3}$ which acts as a curvature reference for surfaces. The Schlaefli conjecture is the origin of the embedding problem for Riemann's geometry. Formally we may write his proposal as

$$
\mathbb{R}(U, V) W=R(U, V) W+\text { extrinsic terms. }
$$

To detail this, we must rewrite this expression in terms of components in a suitable reference frame. This can be done in an arbitrary vielbein defined in the bulk and then by separating the bulk curvature tensor in normal and tangent components. With the purpose of understanding the meaning of the Schlaefli proposal we write these components in the Gaussian frame defined by the embedding map itself. In this frame the components of the extrinsic terms appear as the extrinsic curvature component. This allows us to relate different geometries with different properties through a differential embedding.

In order to make an appropriate embedding between smooth (differentiable) geometries, we use the Nash embedding theorem [14] in order to propose a new theoretical structure able to relate it to a physical theory. Nash showed that any embedded perturbed metric $\widetilde{g}_{\mu \nu}$ can be generated by a continuous sequence of small metric perturbations of a given initially unperturbed geometry $g_{\mu \nu}$ by means of

$$
\tilde{g}_{\mu \nu}=g_{\mu \nu}+\delta y^{a} k_{\mu \nu a}+\delta y^{a} \delta y^{b} g^{\rho \sigma} k_{\mu \rho a} k_{\nu \sigma b}+\cdots
$$

or, equivalently,

$$
k_{\mu \nu}=-\frac{1}{2} \frac{\partial g_{\mu \nu}}{\partial y}
$$

where $y$ is the coordinate related to extra dimensions. Since Nash's smooth deformations are applied to the embedding process, the coordinate $y$ usually noticed in rigid embedded models $[15,16]$ can be omitted in the process for perturbing the element line. This seems particularly interesting to astrophysical and cosmological problems in which traditionally the gravitational perturbation mechanisms are essentially plagued by coordinate gauges due to the group of diffeomorphisms.

The Einstein-Hilbert principle leads to $D$-dimensional Einstein's equations for the bulk metric $\mathscr{G}_{A B}$ in arbitrary coordinates

$$
\mathscr{R}_{A B}-\frac{1}{2} \mathscr{R} \mathscr{G}_{A B}=G_{*} T_{A B}^{*}
$$

where we have dispensed the bulk cosmological constant and $T_{A B}^{*}$ denotes the energy-momentum tensor of the known matter and gauge fields. The constant $G_{*}$ determines the $D$ dimensional energy scale. For the present application, capital Latin indices run from 1 to 5 . Small case Latin indices refer to the only one extra dimension considered. All Greek indices refer to the embedded space-time counting from 1 to 4 .

Concerning the confinement, the four-dimensionality of the space-time is an experimentally established fact associated with the Poincaré invariance of Maxwell's equations and their dualities, also valid for Yang-Mills gauge fields restricted to four dimensions $[17,18]$. Even though the duality properties can be mathematically extended to higher dimensions, we adopt it as a condition based on experimental backgrounds [19]. Therefore, all matter, which interacts with these gauge fields, must for consistency be also defined in the fourdimensional space-time. This consideration complements a physical interpretation for the Nash theorem that provides an interesting mechanism for perturbing and creating new geometries. In this five-dimensional framework present here, only the components that access higher dimensions are related to the extrinsic curvature $k_{\mu \nu}$, while the metric components are confined to the geometry as shown in (2). On the other hand, in spite of all efforts made so far, the gravitational interaction has failed to fit into a similar gauge scheme, so that the gravitational field does not necessarily have the same four-dimensional limitations, regardless of the location of its sources.

In order to recover Einstein's gravity by reversing the embedding, the confinement of ordinary matter and gauge fields implies that the tangent components of $G_{*} T_{A B}^{*}$ in the above equations must coincide with $\left(8 \pi G T_{\mu \nu}\right)$ where $T_{\mu \nu}$ is the energy-momentum tensor of the confined sources. As it may have been already noted, we are essentially reproducing a framework similar to brane-world program [20], with the difference that we apply a dynamical differential embedding and have nothing to do with branes as those defined in string/M theory. Using the Nash embedding theorem together with the four-dimensionality of gauge fields, one can obtain the Einstein-Hilbert principle for the bulk and a $D$-dimensional energy scale $G_{*}$.

In addition, one can define a five-dimensional local embedding with an embedding map $\mathscr{Z}: V_{4} \rightarrow V_{5}$. We admit that the function $\mathscr{Z}^{\mu}$ is a regular and differentiable map with $V_{4}$, which stands for the four embedded space-time types, and $V_{5}$ which refers to the bulk itself. The components 
$\mathscr{Z}^{A}=f^{A}\left(x^{1}, \ldots, x^{4}\right)$ associate with each point of $V_{4}$ a point in $V_{5}$ with coordinates $\mathscr{Z}^{A}$. These coordinates are the components of the tangent vectors of $V_{4}$. Accordingly, calculating the components of (4), one can find the induces equations for the embedded geometry:

$$
\begin{aligned}
R_{\mu \nu}-\frac{1}{2} R g_{\mu \nu}-Q_{\mu \nu} & =-8 \pi G T_{\mu \nu} \\
k_{\mu ; \rho}^{\rho}-h_{, \mu} & =0
\end{aligned}
$$

where now $T_{\mu \nu}$ is the energy-momentum tensor of the confined matter. The quantities $h^{2}=h \cdot h$ with $h=g^{\mu \nu} k_{\mu \nu}$ and $K^{2}=k^{\mu \nu} k_{\mu \nu}$ are the mean curvature and Gaussian curvature, respectively. Moreover, one defines

$$
Q_{\mu \nu}=k_{\mu}^{\rho} k_{\rho \nu}-h k_{\mu \nu}-\frac{1}{2}\left(K^{2}-h^{2}\right) g_{\mu \nu} .
$$

This tensor is independently conserved, as it can be directly verified that (semicolon denoting covariant derivative with respect to $g_{\mu \nu}$ )

$$
Q_{; \nu}^{\mu \nu}=0
$$

A detailed derivation of these equations can be found in [2123] and references therein as well as the higher dimensional case. Hereafter, we use a system of unit such that $c=G=1$.

\section{Induced Potential in a Spherically Symmetric Vacuum Solution}

As shown in a previous work [24], we start with the general static spherically symmetric induced metric that can be described by the line element as

$$
d s^{2}=B(r) d t^{2}-A(r) d r^{2}-r^{2} d \theta^{2}-r^{2} \sin ^{2} \theta d \phi^{2},
$$

where we denote the functions $A(r)=A$ and $B(r)=B$. Thus, one can obtain the following components for the Ricci tensor:

$$
\begin{aligned}
& R_{r r}=\frac{B^{\prime \prime}}{2 B}-\frac{1}{4} \frac{B^{\prime}}{B}\left(\frac{A^{\prime}}{A}+\frac{B^{\prime}}{B}\right)-\frac{1}{r} \frac{A^{\prime}}{A}, \\
& R_{\theta \theta}=-1+\frac{r}{2 A}\left(-\frac{A^{\prime}}{A}+\frac{B^{\prime}}{B}\right)+\frac{1}{A}, \\
& R_{\phi \phi}=\sin ^{2} \theta R_{\theta \theta}, \\
& R_{t t}=-\frac{B^{\prime \prime}}{2 A}+\frac{1}{4} \frac{B^{\prime}}{A}\left(\frac{A^{\prime}}{A}+\frac{B^{\prime}}{B}\right)-\frac{1}{r} \frac{B^{\prime}}{A},
\end{aligned}
$$

where we have $d A / d r=A^{\prime}$ and $d B / d r=B^{\prime}$.

From (5), the gravitational-tensor vacuum equations (with $T_{\mu \nu}=0$ ) can be written in alternative form as

$$
R_{\mu \nu}+\frac{1}{2} Q g_{\mu \nu}=Q_{\mu \nu}
$$

where we use the contraction $Q=g^{\mu \nu} Q_{\mu \nu}$.
The general solution of Codazzi equations (see (6)) is given by

$$
k_{\mu \nu}=f_{\mu} g_{\mu \nu} \quad(\text { no sum on } \mu),
$$

Taking the former equation and the definition of $Q_{\mu \nu}$, one can write

$$
\begin{aligned}
Q_{\mu \nu}= & f_{\mu}^{2} g_{\mu \nu}-\left(\sum_{\alpha} f_{\alpha}\right) f_{\mu} g_{\mu \nu} \\
& -\frac{1}{2}\left(\sum_{\alpha} f_{\alpha}^{2}-\left(\sum_{\alpha} f_{\alpha}\right)^{2}\right) g_{\mu \nu},
\end{aligned}
$$

where

$$
U_{\mu}=f_{\mu}^{2}-\left(\sum_{\alpha} f_{\alpha}\right) f_{\mu}-\frac{1}{2}\left(\sum_{\alpha} f_{\alpha}^{2}-\left(\sum_{\alpha} f_{\alpha}\right)^{2}\right) \delta_{\mu}^{\mu} .
$$

Consequently, we can write $Q_{\mu \nu}$ in terms of $f_{\mu}$ as

$$
Q_{\mu \nu}=U_{\mu} g_{\mu \nu} \quad(\text { no sum on } \mu) \text {. }
$$

A straightforward consequence of the homogeneity of Codazzi equations (see (6)) embedded in five dimensions is that the individual arbitrariness of the functions $f_{\mu}$ can be reduced to a unique arbitrary function $\alpha$ that depends on the radial coordinate. Hence, (12) turns to be

$$
k_{\mu \nu}=\alpha(r) g_{\mu \nu}
$$

with $\alpha(r)=\alpha$. With a straightforward calculation, one can obtain the coefficients of the metric as

$$
\begin{aligned}
& B(r)=1+\frac{K}{r}+\frac{9}{r} \int \alpha^{2}(r) r^{2} d r \\
& A(r)=[B(r)]^{-1}=\left[1+\frac{k}{r}+\frac{9}{r} \int \alpha^{2}(r) r^{2} d r\right]^{-1},
\end{aligned}
$$

where $k$ is a constant. In order to constrain this arbitrariness, we look at the characteristics of the extrinsic curvature itself in the asymptotic limit, which matches the requirement to local astrophysical applications. The extrinsic curvature at infinity goes to a flat space obeying the asymptotically conformal flat condition. This can be understood as the following form:

$$
\lim _{r \rightarrow \infty} k_{\mu \nu}=\lim _{r \rightarrow \infty} \alpha(r) \lim _{r \rightarrow \infty} g_{\mu \nu} .
$$

Since the function $\alpha(r)$ must be analytical at infinity, one can write the simplest option

$$
\alpha(r)=\sum_{n=i}^{s} \frac{\sqrt{-\alpha_{0}}}{\gamma^{*} r^{n}}
$$

where the sum is upon all scalar potentials and the indices $i$ and $s$ are real numbers. Since these scalar potentials have their origin in the extrinsic curvature they do not remain confined in the embedded geometry propagating in the extra dimension. The index $n$ represents all the set of scalar fields that fall 
off with $r$-coordinate following the $n$ th-power law decaying. Equation (20) is essentially the representation of the effect of extrinsic curvature leading to a local modification of the space-time without producing umbilical point as expected for a spherical geometry. Depending on the variation of the function $\alpha(r)$ one can have a bent or stretched geometry without ripping off the manifold, and, curiously, in the same notion as pointed by Riemann himself [25]. It was shown that the parameter $\alpha_{0}$ has cosmological magnitude [24, 26]; that is, it does not depend on individual astrophysical properties and has the same units as the Hubble constant. Its modulus is of the order of $0.677 \mathrm{~km} \cdot \mathrm{s}^{-1} \cdot \mathrm{Mpc}^{-1}$. In addition, to keep the right dimension of (20) we have introduced a unitary parameter $\gamma^{*}$ that has the inverse unit of Hubble constant and also establishes the cosmological horizon in (9). Hence, the horizons of (9) can be found when we set $g_{t t}=0$, and one can obtain

$$
\sum_{n=i}^{s} \frac{9 \alpha_{0}}{3-2 n} r^{3-2 n}+r-2 M=0,
$$

which is a polynomial equation of order $(3-2 n)$ that gives a class of horizons to be defined for all allowed values of $n$.

Using (17) and (20), one can obtain an explicit form of the coefficient $B(r)$ given by

$$
B(r)=1+\frac{k\left(9 \alpha_{0}^{2}+1\right)}{r}-\sum_{n=i}^{s} \frac{9 \alpha_{0}}{\gamma^{*}(2 n-3)} r^{2(1-n)} .
$$

In terms of the correspondence principle with Einstein equations, we set $k\left(9 \alpha_{0}^{2}+1\right)=-2 M$, which remains valid even in the limit when $\alpha_{0} \rightarrow 0$ in order to obtain the asymptotically flat solution. It is worth mentioning a qualitative aspect that the diffeomorphic transformations commonly known in the realm of general relativity do not necessarily apply even when $\alpha_{0} \rightarrow 0$. This comes from the fact that structure of the embedded space (and its relation to the bulk) breaks down the equivalence principle of general relativity. In other words, the diffeomorphism must be imposed as an assumption to recover full general relativity.

\section{Scalar Perturbations}

In this section, we focus our attention on the study of (22) under scalar perturbations. The study of quasinormal modes can be treated by scalar fields and appropriate metrics. In this section, we construct the Klein-Gordon equation in a curved space-time embedded in a five-dimensional space-time. In order to achieve such a goal, we consider the line element in the form

$$
d s^{2}=f(r) d t^{2}-f(r)^{-1} d r^{2}+r^{2} d \Omega .
$$

It is well known that the massless Klein-Gordon equation is given by

$$
\nabla_{\mu} \nabla^{\mu} \Phi=0
$$

or, equivalently,

$$
\frac{1}{\sqrt{-g}} \partial_{\mu}\left(\sqrt{-g} g^{\mu \nu} \partial_{\mu} \Phi\right)=0,
$$

where $\Phi=\Phi(r, \theta, \phi, t)$. Using (23), we can write

$$
\sqrt{-g}=r^{2} \sin \theta
$$

Moreover, (25) can be written as

$$
\begin{aligned}
\frac{1}{f(r)} & \frac{\partial^{2} \Phi}{\partial t^{2}}-\frac{\partial}{\partial r}\left(f(r) \frac{\partial \Phi}{\partial r}\right)-\frac{2 f(r)}{r} \frac{\partial \Phi}{\partial r} \\
& -\frac{1}{r^{2} \sin \theta} \frac{\partial}{\partial \theta}\left(\sin \theta \frac{\partial \Phi}{\partial \phi}\right)-\frac{1}{r^{2} \sin ^{2} \theta} \frac{\partial^{2} \Phi}{\partial \phi^{2}}=0 .
\end{aligned}
$$

Taking the ansatz

$$
\Phi(r, \theta, \phi, t)=\sum_{l=0}^{\infty} \sum_{m=-l}^{l} Y_{l, m}(\theta, \phi) e^{-i \omega t} \frac{\psi_{l, m}(r)}{r},
$$

we obtain

$$
\begin{aligned}
& {\left[\frac{1}{\sin \theta} \frac{\partial}{\partial \theta}\left(\sin \theta \frac{\partial}{\partial \phi}\right)-\frac{1}{\sin ^{2} \theta} \frac{\partial^{2}}{\partial \phi^{2}}\right] Y_{l, m}(\theta, \phi)} \\
& \quad=-l(l+1) Y_{l, m}(\theta, \phi),
\end{aligned}
$$

where $Y_{l, m}(\theta, \phi)$ are the spherical harmonics.

$$
\begin{gathered}
\left(\frac{-\omega^{2}}{f(r)}+\frac{l(l+1)}{r^{2}}-f(r) \frac{\partial^{2}}{\partial r^{2}}-f^{\prime}(r) \frac{\partial}{\partial r}\right. \\
\left.-\frac{2 f(r)}{r} \frac{\partial}{\partial r}\right) \frac{\psi_{l, m}(r)}{r}=0 .
\end{gathered}
$$

Taking the following coordinates change $d x=d r / f(r)$, we obtain the master wave equation

$$
\frac{d^{2} \psi_{l, m}(r)}{d x^{2}}+\left(\omega^{2}-V(x)\right) \psi_{l, m}(r)=0
$$

where we denote

$$
V(x)=f(x) \frac{l(l+1)}{x^{2}}+f(x) \frac{f^{\prime}(x)}{x} .
$$

If we consider (22), then we finally get

$$
f(r)=1-\frac{2 M}{r}+\sum_{j} \frac{\alpha_{0}}{2 j-3} r^{2(1-j)} .
$$

Once the potential $V$ is well defined, we plot its behaviour for different values of $\alpha_{0}$ and $l$, which are given in Figures 1 and 2.

For a massive field the potential is modified to $V(r) \rightarrow$ $V(r)+\mu^{2} f(r) / r$; the angular part remains the same. In Figure 3 we plot such a potential for $\alpha_{0}=0.677, M=1$, and $\mu=0.1 M$.

It is interesting to analyze the potential as a function of the tortoise coordinate to understand the black hole stability. Using such a coordinate the space is described from the black hole horizon to infinity. The event horizon is calculated as a solution of (21) which yields $r_{h}=0.459413$ for $\alpha=0.677$ 


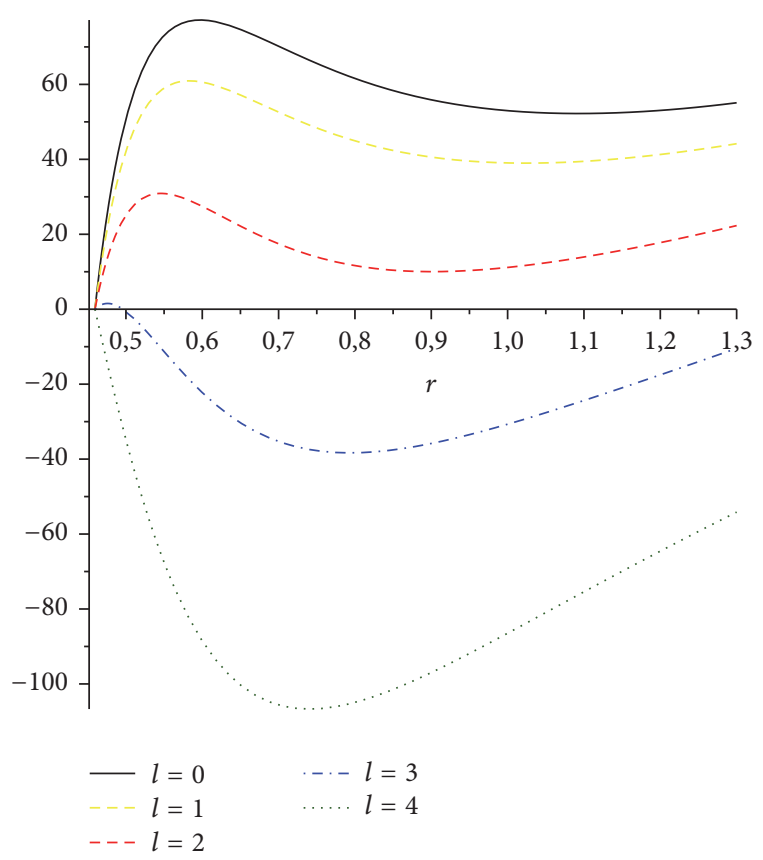

FIgURE 1: The potential $V$ in function of the radius for values $l=0$ (solid thick line), $l=1$ (long-dashed line), $l=2$ (dashed line), $l=3$ (dot-dashed line), and $l=4$ (dotted line) for the fixed values of $\alpha_{0}=$ $0.677, M=1$, and $\mu=0$.

and $r_{h}=0.534067$ for $\alpha=2$. It was argued by Chandrasekhar [27] that the stability of the perturbation is determined by the sign of the potential outside the black hole. As it is seen from Figures 4,5 , and 6 , the potential seems to be unstable for high values of $l$ since it is negative; particularly for Figure 6 the potential stays positive outside the black hole, which could change its sign for bigger values of $l$. This justifies further investigation; thus we will present in the next section the calculation of the quasinormal modes.

For the sake of completeness we present the behaviour of the potential in the whole space-time outside the black hole (from $r_{h}=0.459413$ to $\infty$ ) for $\alpha_{0}=0.677$ and $\mu=0, \mu=$ $0.1 M$, respectively, in Figures 7 and 8 .

For $\alpha_{0}=2$ the potential is too narrow to be seen in the range 0.534067 to $\infty$. Such a behaviour is expected due to cosmological implications of the constant $\alpha_{0}$. For instance, the magnitude of this parameter would lead to a big rip model.

\section{Quasinormal Modes in WKB Approximation}

In this section, we use the Wentzel, Kramers, and Brillouin $(\mathrm{WKB})$ approximation in such a regime where the frequency is given by [28]

$$
\frac{\imath\left(\omega^{2}-V_{0}\right)}{\sqrt{-2 V_{0}^{\prime \prime}}}-\sum_{i=2}^{k} \Lambda_{i}=n+\frac{1}{2},
$$

where $V_{0}$ and $V_{0}^{\prime \prime}$ are the effective potential and its second derivative, respectively, as taken at the point of the maximum

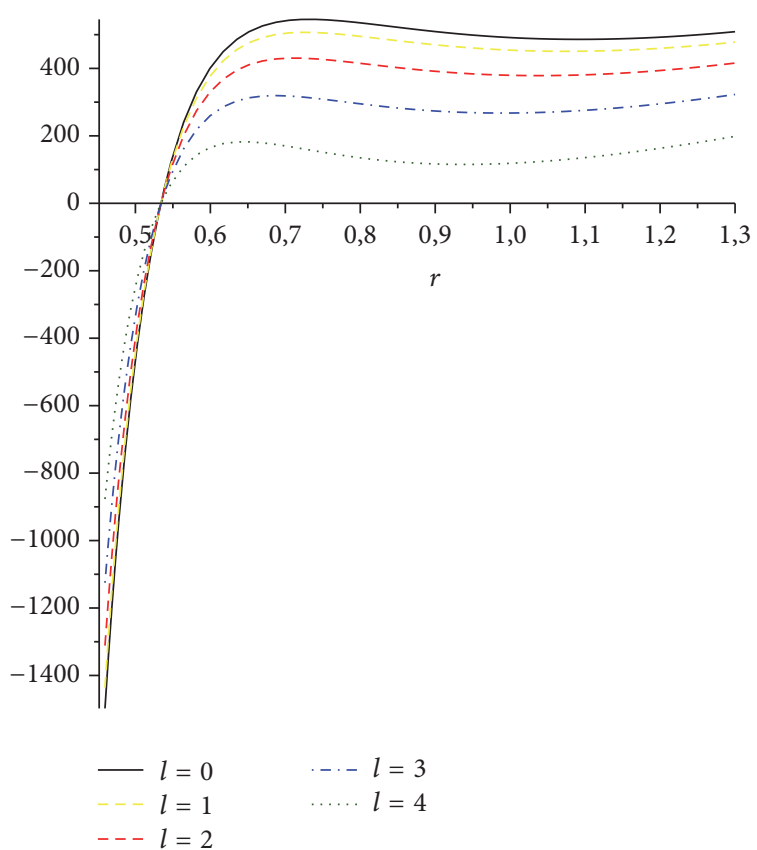

FIgURE 2: The potential $V$ in function of the radius for values $l=0$ (solid thick line), $l=1$ (long-dashed line), $l=2$ (dashed line), $l=3$ (dot-dashed line), and $l=4$ (dotted line) for the fixed values of $\alpha_{0}=$ $2, M=1$, and $\mu=0$.

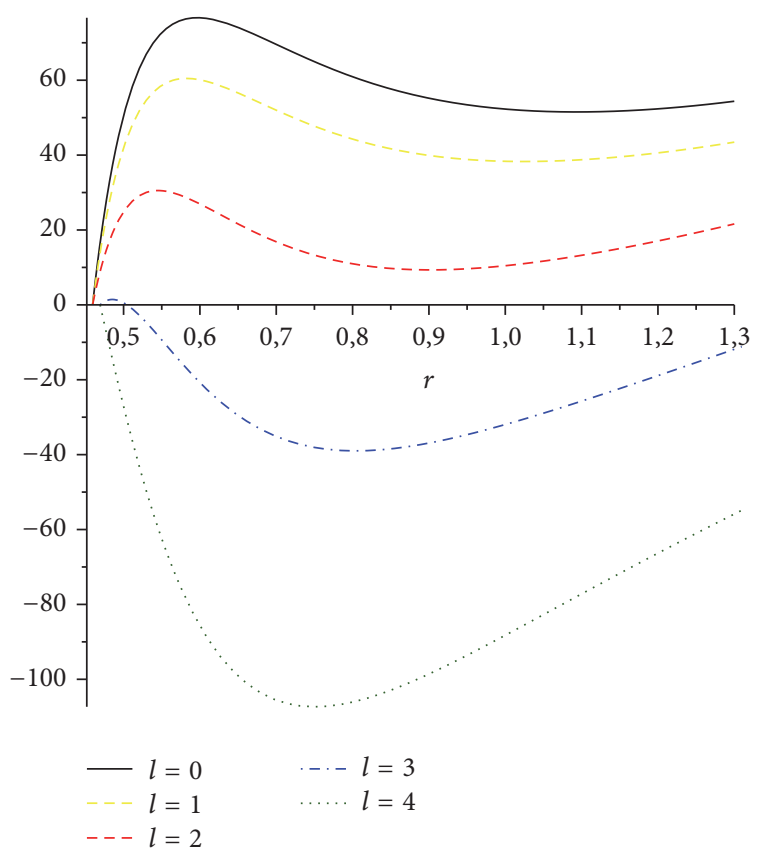

FIgURE 3: The potential $V$ in function of the radius for values $l=0$ (solid thick line), $l=1$ (long-dashed line), $l=2$ (dashed line), $l=3$ (dot-dashed line), and $l=4$ (dotted line) for the fixed values of $\alpha_{0}=$ $0.677, M=1$, and $\mu=0.1 M$.

of $V$. The sum above represents high order correction in the usual WKB method [29]. Thus the higher the order of approximation is, the better the result is, which could be 


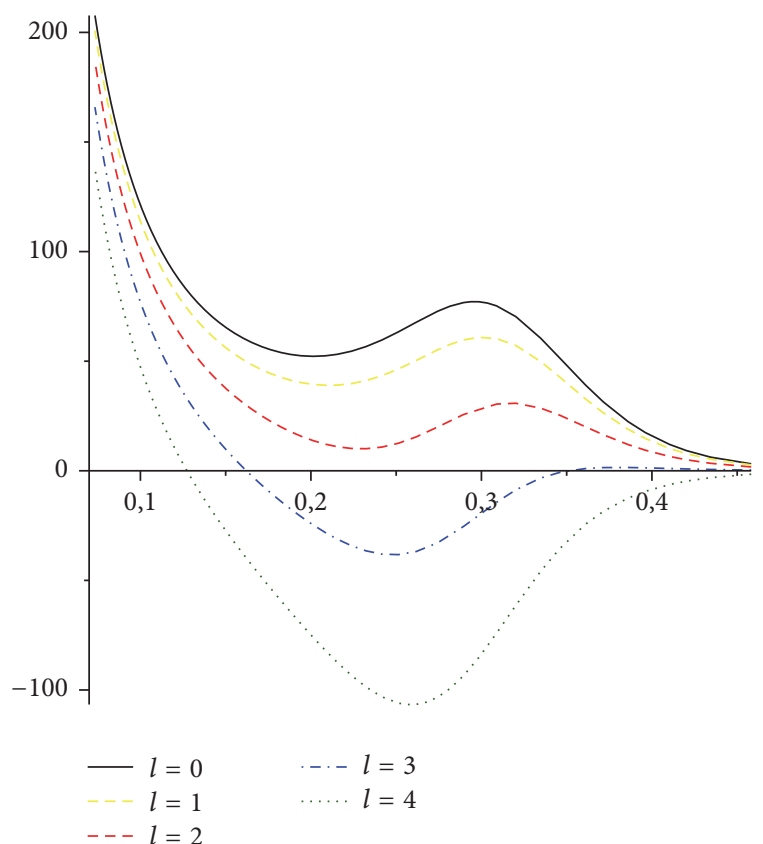

Figure 4: The potential $V$ in function of the tortoise coordinates for the fixed values of $\alpha_{0}=0.677, M=1$, and $\mu=0$.

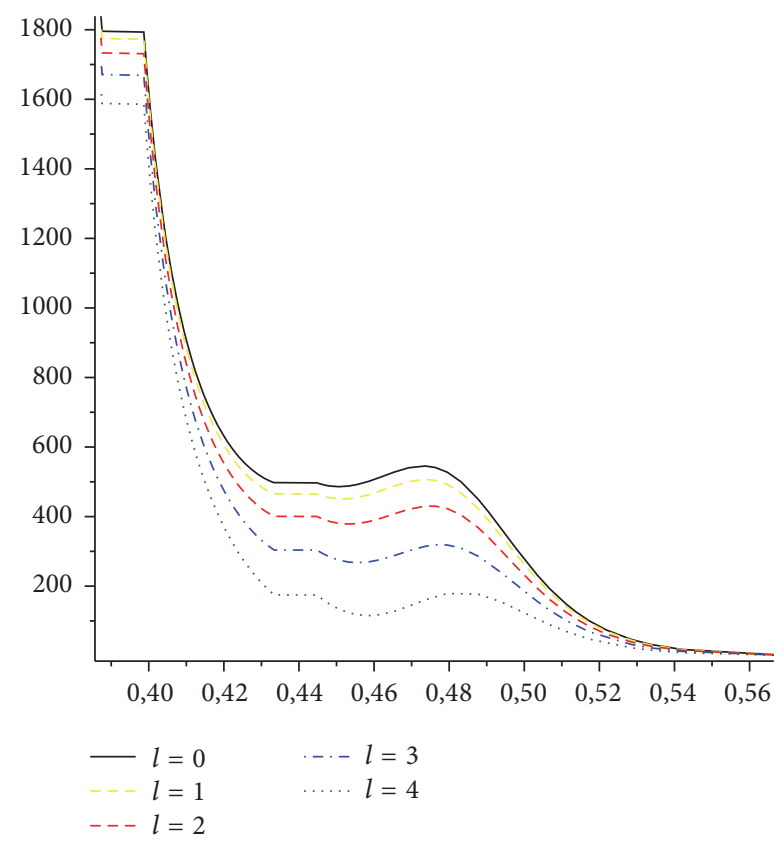

FIgURE 5: The potential $V$ in function of the tortoise coordinates for the fixed values of $\alpha_{0}=2, M=1$, and $\mu=0$.

comparable to numerical methods [28]. In this sense it is enough to work with the third-order WKB approximation [30], which is given by

$$
\begin{aligned}
\omega_{n, l}^{2}= & {\left[V_{0}+\left(-2 V_{0}^{\prime \prime}\right)^{1 / 2} \Lambda\right] } \\
& -\imath\left(n+\frac{1}{2}\right)\left(-2 V_{0}^{\prime \prime}\right)^{1 / 2}(1+\Omega),
\end{aligned}
$$

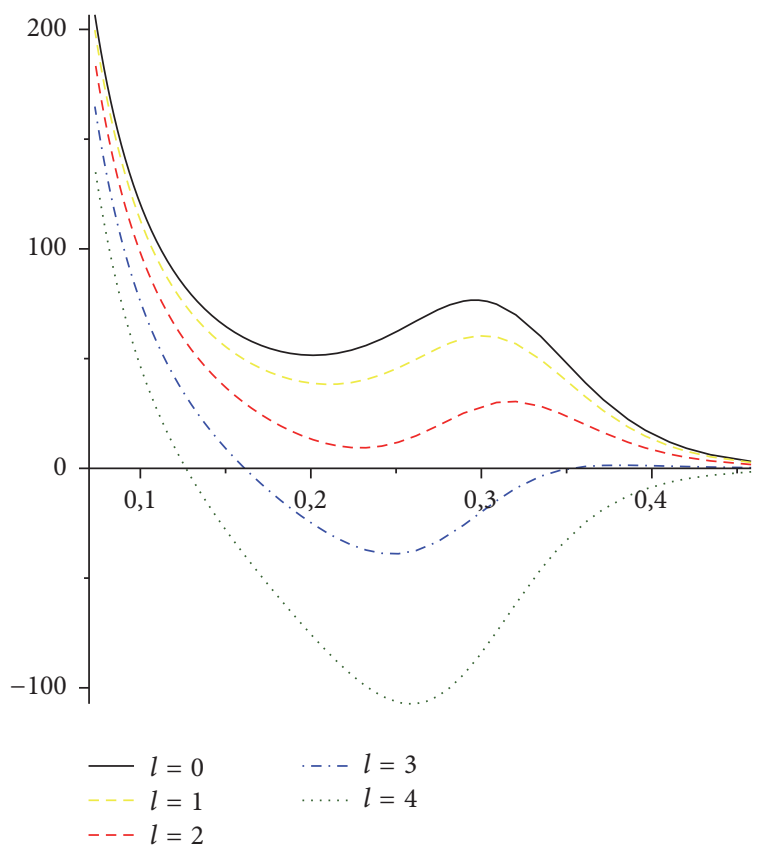

FIgURE 6: The potential $V$ in function of the tortoise coordinates for the fixed values of $\alpha_{0}=0.677, M=1$, and $\mu=0.1 M$.

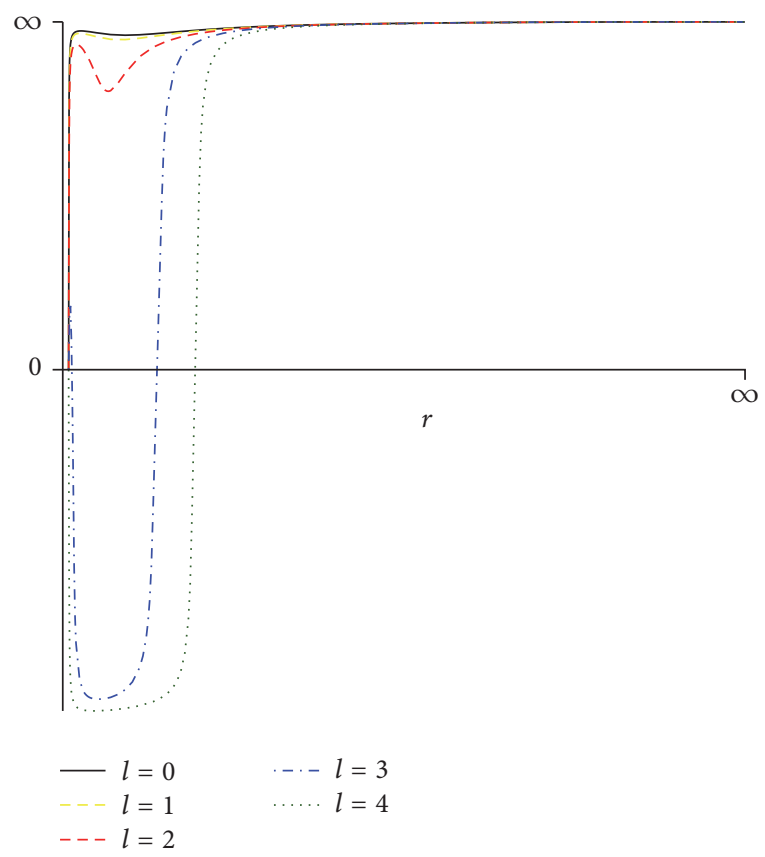

FIgURE 7: The potential $V$ in function of $r$, from $r_{h}=0.459413$ to $\infty$ for $\alpha_{0}=0.677, M=1$, and $\mu=0$.

where

$$
\begin{aligned}
\Lambda & =\frac{1}{\left(-2 V_{0}^{\prime \prime}\right)^{1 / 2}}\left[\frac{1}{8}\left(\frac{V_{0}^{(4)}}{V_{0}^{\prime \prime}}\right)\left(\frac{1}{4}+\beta^{2}\right)\right. \\
& \left.-\frac{1}{288}\left(\frac{V_{0}^{\prime \prime \prime}}{V_{0}^{\prime \prime}}\right)^{2}\left(7+60 \beta^{2}\right)\right],
\end{aligned}
$$




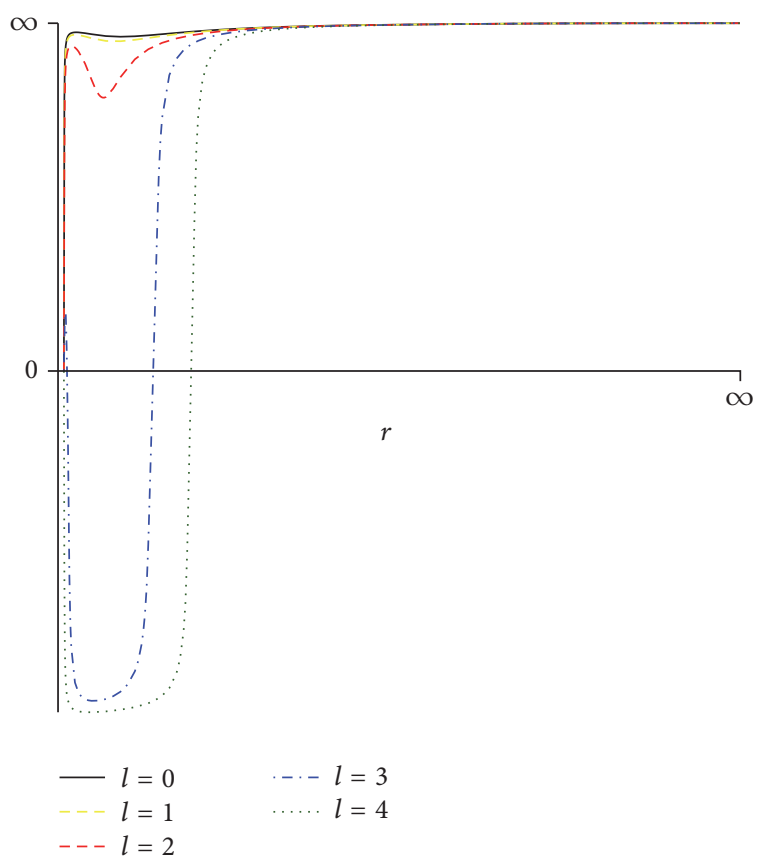

FIgURE 8: The potential $V$ in function of $r$, from $r_{h}=0.459413$ to $\infty$ for $\alpha_{0}=0.677, M=1$, and $\mu=0.1 M$.

$$
\begin{aligned}
\Omega & =\frac{1}{\left(-2 V_{0}^{\prime \prime}\right)}\left[\frac{5}{6912}\left(\frac{V_{0}^{\prime \prime \prime}}{V_{0}^{\prime \prime}}\right)^{4}\left(77+188 \beta^{2}\right)\right. \\
& -\frac{1}{384}\left(\frac{\left(V_{0}^{\prime \prime \prime}\right)^{2} V_{0}^{(4)}}{\left(V_{0}^{\prime \prime}\right)^{3}}\right)\left(51+100 \beta^{2}\right) \\
& +\frac{1}{2304}\left(\frac{V_{0}^{(4)}}{V_{0}^{\prime \prime}}\right)^{2}\left(67+68 \beta^{2}\right) \\
& +\frac{1}{288}\left(\frac{V_{0}^{\prime \prime \prime} V_{0}^{(5)}}{\left(V_{0}^{\prime \prime}\right)^{2}}\right)\left(19+28 \beta^{2}\right) \\
& \left.-\frac{1}{288}\left(\frac{V_{0}^{(6)}}{V_{0}^{\prime \prime}}\right)\left(5+4 \beta^{2}\right)\right] .
\end{aligned}
$$

Here $\beta=n+1 / 2$ and $V_{0}^{(n)}=\left.\left(d^{n} V / d x^{n}\right)\right|_{x=x_{0}}$. The point $x_{0}$ is the solution of the equation $(d V / d x)\left(x_{0}\right)=0$, which means a point of maximum of the effective potential. We point out that $x$ is the tortoise coordinate, already defined.

Next we chart our results on Tables 1, 2, and 3. We see from Table 1 that the scalar perturbation for a realistic value of $\alpha$ is not stable. This complements our previous work in which we concluded that such a solution is not globally thermodynamically stable and its stability is reduced to very constrained range [31]. On the other hand from Tables 2 and 3 we see that the stability may be achieved by a choice of a hypothetical value of $\alpha$ (in this case, $\alpha>0.667$ will lead to a faster accelerated regime as expected for a phantom-like
TABLE 1: Quasinormal modes of scalar perturbations for $\alpha_{0}=0.677$ with $M=1$ and $\mu=0$.

\begin{tabular}{llc}
\hline$l$ & $n$ & $\omega_{n l}$ \\
\hline 0 & 0 & $5.102614010-8.026857534 i$ \\
1 & 0 & $4.444548318-8.863420102 i$ \\
& 1 & $11.59600413-26.25141968 i$ \\
& 0 & $2.556070877-9.893346520 i$ \\
& 1 & $6.342841226-24.95915206 i$ \\
3 & 2 & $12.94011467-41.62859430 i$ \\
& 0 & $0.3142845589+12.96051935 i$ \\
& 1 & $38.84486810-53.32057992 i$ \\
& 2 & $93.40199364-111.3133472 i$ \\
4 & 3 & $160.5504266-181.4993689 i$ \\
& 0 & $2.789319410+26.91329506 i$ \\
& 1 & $136.9620887+165.7373689 i$ \\
& 2 & $317.9204012+354.6421808 i$ \\
& 3 & $540.1554148+583.4781202 i$ \\
& 4 & $797.3633624+846.4289944 i$ \\
& 0 & $6.400241451+55.35392070 i$ \\
& 1 & $301.0022385+362.7954640 i$ \\
& 2 & $697.9325995+777.2504936 i$ \\
& 3 & $1185.464821+1279.187469 i$ \\
& 4 & $1749.721283+1855.936081 i$ \\
\hline
\end{tabular}

TABLE 2: Quasinormal modes of scalar perturbations for $\alpha_{0}=2$ with $M=1$ and $\mu=0$.

\begin{tabular}{llc}
\hline$l$ & $n$ & $\omega_{n l}$ \\
\hline 0 & 0 & $28.54240977-35.52756231 i$ \\
1 & 0 & $28.98497167-36.65093824 i$ \\
& 1 & $103.8128126-126.3594331 i$ \\
2 & 0 & $29.20727668-38.11077359 i$ \\
& 1 & $106.1438323-128.6137680 i$ \\
& 2 & $224.2314700-254.7665296 i$ \\
3 & 0 & $26.35460874-37.16001393 i$ \\
& 1 & $95.10645314-118.6073793 i$ \\
& 2 & $200.6518286-232.2842502 i$ \\
4 & 3 & $333.1889473-371.1040372 i$ \\
& 0 & $14.72079936-30.28374333 i$ \\
& 1 & $47.53015516-80.58865151 i$ \\
& 2 & $100.4540639-146.2752812 i$ \\
& 3 & $169.1566913-224.7764688 i$ \\
& 4 & $250.6229786-314.4239174 i$ \\
& 0 & $0.5248454700+28.40271014 i$ \\
& 1 & $45.08904231-85.15794051 i$ \\
& 2 & $116.4495623-166.1450658 i$ \\
& 3 & $205.7296676-263.8287746 i$ \\
& & $309.9693513-375.5150908 i$ \\
\hline
\end{tabular}

cosmology [26] and black holes in this context are usually unstable) or for a massive scalar field. In all scenarios the real part of the frequency increases rapidly, which indicates that the quasinormal frequency oscillates faster for each mode. 
TABLE 3: Quasinormal modes of massive scalar perturbations for $\alpha_{0}=0.677$ and $\mu=0.1 M$ with $M=1$.

\begin{tabular}{llc}
\hline$l$ & $n$ & $\omega_{n l}$ \\
\hline 0 & 0 & $5.102614010-8.026857534 i$ \\
1 & 0 & $29.00936098-36.67332200 i$ \\
& 1 & $103.9107237-126.4501766 i$ \\
& 0 & $29.23683246-38.13734008 i$ \\
& 1 & $106.2610002-128.7222323 i$ \\
3 & 2 & $224.4751039-254.9976125 i$ \\
& 0 & $26.38849620-37.18864399 i$ \\
& 1 & $95.24043132-118.7279173 i$ \\
& 2 & $200.9301766-232.5433005 i$ \\
4 & 3 & $333.6438082-371.5352605 i$ \\
& 0 & $14.74857813-30.30037176 i$ \\
& 1 & $47.63840864-80.66160380 i$ \\
& 2 & $100.6803470-146.4444515 i$ \\
& 3 & $169.5250565-225.0711954 i$ \\
5 & 4 & $797.6039393+846.6872620 i$ \\
& 0 & $0.5217309759+28.39953701 i$ \\
& 1 & $45.02864470-85.11181068 i$ \\
& 2 & $116.3098696-166.0264653 i$ \\
& 3 & $205.4963229-263.6210918 i$ \\
& 4 & $309.6292522-375.2043911 i$ \\
\hline
\end{tabular}

Hence, due to the positive growing of the imaginary part, such a behaviour is very similar to a resonance.

\section{Conclusion}

In this article we have analyzed the stability of the line element obtained in [24, 31], under scalar perturbations. Such a metric was derived by searching a solution for a four-dimensional metric spherically symmetric embedded in a five-dimensional bulk space. Thus we have identified an effective potential that composes the gravitational field equations. We have calculated the scalar quasinormal modes of Klein-Gordon equation in order to get some information on the stability of our metric. We have concluded that the massive scalar perturbation yields a stable configuration or a nonrealistic massless perturbation, as can be possibly seen from Tables 2 and 3. We point out that the instability of the solution for massless scalar perturbation with $\alpha_{0}=0.677$ complements our previous observation about the thermodynamical features of such a solution that leads to a local thermal stability of those black holes constrained to a narrow window of scalar fields [31]. A natural extension of this work is to study a different type of perturbations (e.g., vector, electromagnetic, gravitational) in order to perform a more general analysis on stability and possible orbits.

Interestingly, only considering the scalar modes, the resulting frequencies present high absolute values of the high overtone modes, differently as compared to those of rigid embedding models [32-35]. It has a dramatic consequence that leads to resonant-like black holes to restricted spectra of scalar modes. All presented results suggest that the physical consequence of a four-sphere symmetric metric dynamically embedded in five-dimensional bulk may lead to unstable solutions and evinces that a higher dimensional structure is a necessary condition. It reinforces, for example, why RS solutions for a Schwarzschild back hole may be unstable [36].

Also, another interesting fact emerges from the negative gap in the effective potential in the present model as it matches some results known in literature. For instance, in the context of a generalised general relativity, the Einstein-GaussBonnet theory (also known as Lovelock theory) [37] admits static symmetric black holes but they are unstable for five and six dimensions in which the hyperbolic equations do not arise from Cauchy initial data. Specifically, the scalar perturbations in five dimensions provide a considerable negative effective potential where the hyperbolicity is violated regarding small black holes (with arbitrary small mass) and they do not exist.

Similar conclusions are found in [38] that show instability of Einstein-Gauss-Bonnet black holes in de Sitter space-time. This instability has its origin in the nonzero positive cosmological constant through the so-called eikonal instability (in the sense of geometrical optics the quasinormal modes go to infinity) which leads to instability with agreement that "unstable modes cannot be oscillatory when perturbing spherically symmetric static black holes" [39], which reinforces our results in a different context. The main point we want to stress is that such instability suggested by our analysis comes from the fact that the five-dimensional bulk is not appropriate geometric architecture for a dynamical embedding concerning static spherical symmetries [40-42] and the stable points should be very restricted [31]; thus it may represent a serious constraint for five-dimensional embedding models even though global embedding may be possible [43].

Moreover, we do not have specifically a solution directly related to RS models but using the WKB method our results show high absolute values in both low and high overtones, differently from those presented in the RS models. As a counterexample, we need to show what happens in this particular case, as one can see this behaviour essentially at the mode $l=3$ with $\alpha_{0}=0.667$, which shows being sensitive to scalar perturbations. We point out that the effective potentials in higher dimensions are commonly nonpositive definite, which may cause instability, so in a way we expected negative values of the effective potential at some point. Remarkably, we also expected that when the parameter $\alpha_{0}>0.667$ the dynamics of the embedded space-time should resemble one that phantom (exotic) models have due to the fact that black holes from this sort of behaviour are generally unstable, but we had, in principle, positive values for the effective potential, where in some sense we should expect some region of stability for this condition. On the other hand, the importance of the values shown by using the WKB method shows us that, in summary, the spherically neutral symmetric metric provides resonant-like black holes showing that they are sensitive to scalar perturbations and may be stable at a low range of set of scalar fields. This result is a consequence of the type of embedding made (e.g., Schwarzschild metric is not embedded in five dimensions). In other words, it is necessary to add more degrees of freedom leading to a six-dimensional 
space-time, if one thinks in terms of a sought-after future unified field theory.

\section{Competing Interests}

The authors declare that they have no competing interests.

\section{References}

[1] E. Berti, V. Cardoso, and J. P. S. Lemos, "Quasinormal modes and classical wave propagation in analogue black holes," Physical Review D, vol. 70, no. 12, Article ID 124006, 2004.

[2] T. Regge and J. A. Wheeler, "Stability of a schwarzschild singularity," Physical Review, vol. 108, no. 4, p. 1063, 1957.

[3] C. V. Vishveshwara, "Scattering of gravitational radiation by a schwarzschild black-hole," Nature, vol. 227, no. 5261, pp. 936938, 1970.

[4] R. A. Konoplya, "Gravitational quasinormal radiation of higherdimensional black holes," Physical Review D, vol. 68, no. 12, Article ID 124017, 2003.

[5] K. Martel and E. Poisson, "Gravitational perturbations of the Schwarzschild spacetime: a practical covariant and gaugeinvariant formalism," Physical Review D, vol. 71, no. 10, Article ID 104003, 2005.

[6] A. S. Miranda and V. T. Zanchin, "Quasinormal modes of planesymmetric anti-de Sitter black holes: a complete analysis of the gravitational perturbations," Physical Review. D. Third Series, vol. 73, no. 6, 064034, 14 pages, 2006.

[7] H. Kodama, A. Ishibashi, and O. Seto, "Brane world cosmology: gauge-invariant formalism for perturbation," Physical Review D, vol. 62, no. 6, Article ID 064022, 2000.

[8] J. Maldacena, "The large $N$ limit of superconformal field theories and supergravity," Advances in Theoretical and Mathematical Physics, vol. 2, no. 2, pp. 231-252, 1998.

[9] B. P. Abbott, R. Abbott, T. D. Abbott et al., "Observation of gravitational waves from a binary black hole merger," Physical Review Letters, vol. 116, no. 6, Article ID 061102, 2016.

[10] R. Konoplya and A. Zhidenko, "Detection of gravitational waves from black holes: is there a window for alternative theories?" Physics Letters B, vol. 756, pp. 350-353, 2016.

[11] N. Yunes, K. Yagi, and F. Pretorius, "Theoretical physics implications of the binary black-hole mergers GW150914 and GW151226," Physical Review D, vol. 94, no. 8, Article ID 084002, 2016.

[12] G. Perelman, "Finite extinction time for the solutions to the ricci flow on certain three-manifolds," Topologica, vol. 1, no. 1, p. 005, 2008.

[13] G. Perelman, "The entropy formula for the Ricci flow and its geometric applications," https://arxiv.org/abs/math/0211159.

[14] J. Nash, "The imbedding problem for riemannian manifolds," The Annals of Mathematics, vol. 63, no. 1, pp. 20-63, 1956.

[15] L. Randall and R. Sundrum, "Large mass hierarchy from a small extra dimension," Physical Review Letters, vol. 83, no. 17, pp. 3370-3373, 1999.

[16] L. Randall and R. Sundrum, "An alternative to compactification," Physical Review Letters, vol. 83, no. 23, pp. 4690-4693, 1999.

[17] S. K. Donaldson, "Smooth 4-manifolds with definite intersection form," Contemporary Mathematics (AMS), vol. 35, p. 201, 1984.
[18] C. H. Taubes, "An introduction to self-dual connections," Contemporary Mathematics (AMS), vol. 35, p. 493, 1984.

[19] C. S. Lim, "The Higgs particle and higher-dimensional theories," Progress of Theoretical and Experimental Physics, vol. 2014, no. 2, Article ID 02A101, 2014.

[20] N. Arkani-Hamed, S. Dimopoulosb, and G. Dvalic, "The hierarchy problem and new dimensions at a millimeter," Physics Letters B, vol. 429, no. 3-4, pp. 263-272, 1998.

[21] M. D. Maia, E. M. Monte, J. M. F. Maia, and J. S. Alcaniz, "On the geometry of dark energy," Classical and Quantum Gravity, vol. 22, no. 9, pp. 1623-1636, 2005.

[22] M. D. Maia, A. J. S. Capistrano, J. S. Alcaniz, and E. M. Monte, "The deformable universe," General Relativity and Gravitation, vol. 43, no. 10, pp. 2685-2700, 2011.

[23] M. D. Maia, N. Silva, and M. C. B. Fernandes, "Brane-world quantum gravity," Journal of High Energy Physics, vol. 2007, no. 4, article 047, 2007.

[24] A. J. S. Capistrano and L. A. Cabral, "Geometrical aspects on the dark matter problem," Annals of Physics, vol. 348, pp. 64-83, 2014.

[25] B. Riemann, "On the Hypothesis That Lie at the Foundations of Geometry (1854), translated by W. K. Cli ord," Nature, vol. 8 , article 114, 1873, See also comments in P. Pesic, Beyond Geometry, Dover, 2007.

[26] A. J. S. Capistrano, "Constraints on cosmokinetics of smooth deformations," Monthly Notices of the Royal Astronomical Society, vol. 448, no. 2, pp. 1232-1239, 2015.

[27] S. Chandrasekhar, The Mathematical Theory of Black Holes, Oxford University Press, 1992.

[28] R. A. Konoplya and A. Zhidenko, "Quasinormal modes of black holes: from astrophysics to string theory," Reviews of Modern Physics, vol. 83, no. 3, pp. 793-836, 2011.

[29] R. A. Konoplya, "Gravitational quasinormal radiation of higherdimensional black holes," Physical Review. D. Third Series, vol. 68, no. 12, 124017, 7 pages, 2003.

[30] S. Iyer, "Black-hole normal modes: a WKB approach. II. Schwarzschild black holes," Physical Review D, vol. 35, no. 12, pp. 3632-3636, 1987.

[31] A. J. S. Capistrano, A. C. Gutiérrez-Piñeres, S. C. Ulhoa, and R. G. G. Amorim, "On the thermal stability of a static spherically symmetric black holes in Nash embedding framework," https://arxiv.org/abs/1507.06664v4.

[32] E. Abdalla, B. Cuadros-Melgara, A. B. Pavana, and C. Molinab, "Stability and thermodynamics of brane black holes," Nuclear Physics B, vol. 752, no. 1-2, pp. 40-59, 2006.

[33] E. Berti, K. D. Kokkotas, E. Papantonopoulos et al., "Stability of five-dimensional rotating black holes projected on the brane," Physical Review D, vol. 68, no. 6, Article ID 064020, 2003.

[34] P. Kanti and R. A. Konoplya, "Quasinormal modes of branelocalized standard model fields," Physical Review D, vol. 73, no. 4, Article ID 044002, 2006.

[35] P. Kanti, R. A. Konoplya, and A. Zhidenko, "Quasinormal modes of brane-localized standard model fields. II. Kerr black holes," Physical Review D, vol. 74, no. 6, Article ID 064008, 2006.

[36] H. Yoshino, "On the existence of a static black hole on a brane," Journal of High Energy Physics, vol. 2009, 2009.

[37] G. Papallo and H. S. Reall, "Graviton time delay and a speed limit for small black holes in Einstein-Gauss-Bonnet theory," Journal of High Energy Physics, vol. 2015, no. 11, article 109, 2015. 
[38] M. A. Cuyubamba, R. A. Konoplya, and A. Zhidenko, "Quasinormal modes and a new instability of Einstein-Gauss-Bonnet black holes in the de Sitter world," Physical Review D, vol. 93, Article ID 104053, 2016.

[39] R. A. Konoplya, K. Murata, J. Soda, and A. Zhidenko, "Looking at the Gregory-Laflamme instability through quasinormal modes," Physical Review D. Particles, Fields, Gravitation, and Cosmology, vol. 78, no. 8, Article ID 084012, 2008.

[40] S. A. Paston and A. A. Sheykin, "Embeddings for the Schwarzschild metric: classification and new results," Classical and Quantum Gravity, vol. 29, no. 9, Article ID 095022, 2012.

[41] E. M. Monte, "The embedding of Schwarzschild in Braneworld," International Journal of Theoretical Physics, vol. 48, no. 2, pp. 409-413, 2009.

[42] M. P. Dabrowski, "Isometric embedding of the spherically symmetric Stephani universe: some explicit examples," Journal of Mathematical Physics, vol. 34, no. 4, pp. 1447-1479, 1993.

[43] A. A. Sheykin and S. A. Paston, "Classification of minimum global embeddings for nonrotating black holes," Theoretical and Mathematical Physics, vol. 185, no. 1, pp. 1547-1556, 2015. 

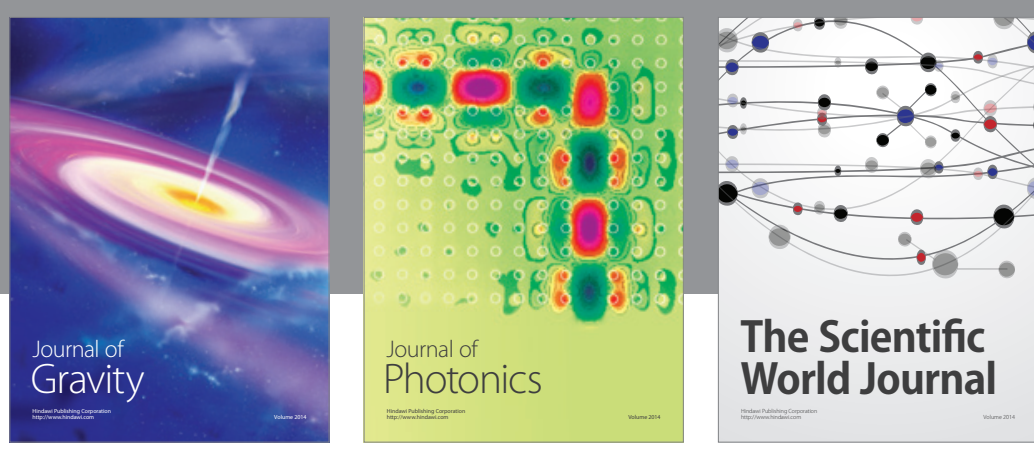

The Scientific World Journal
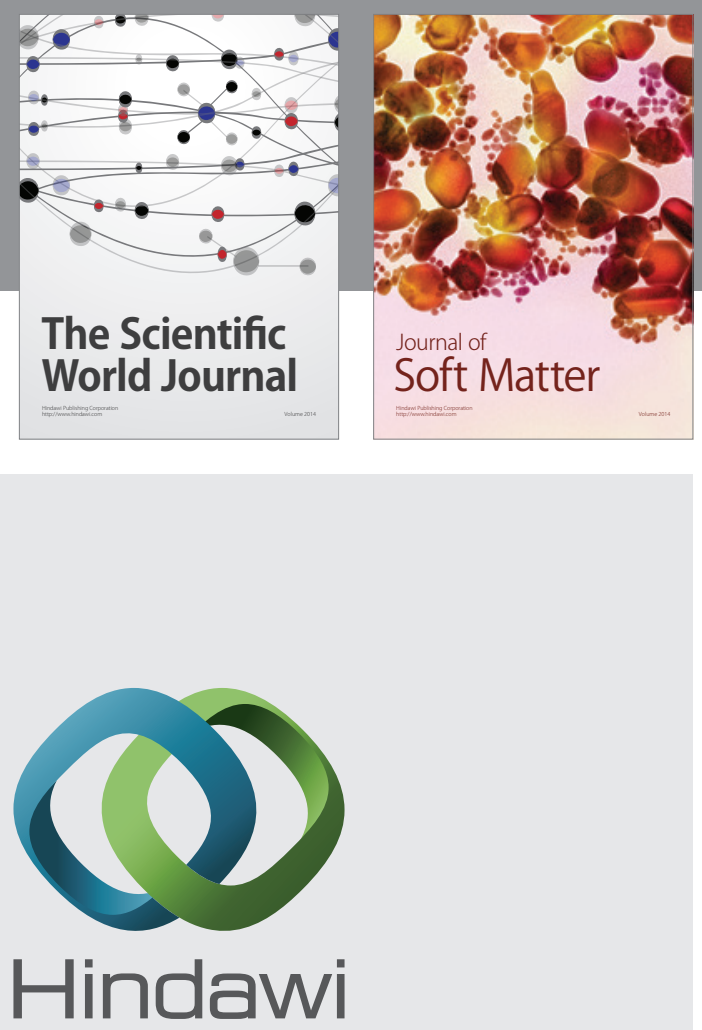

Submit your manuscripts at

https://www.hindawi.com
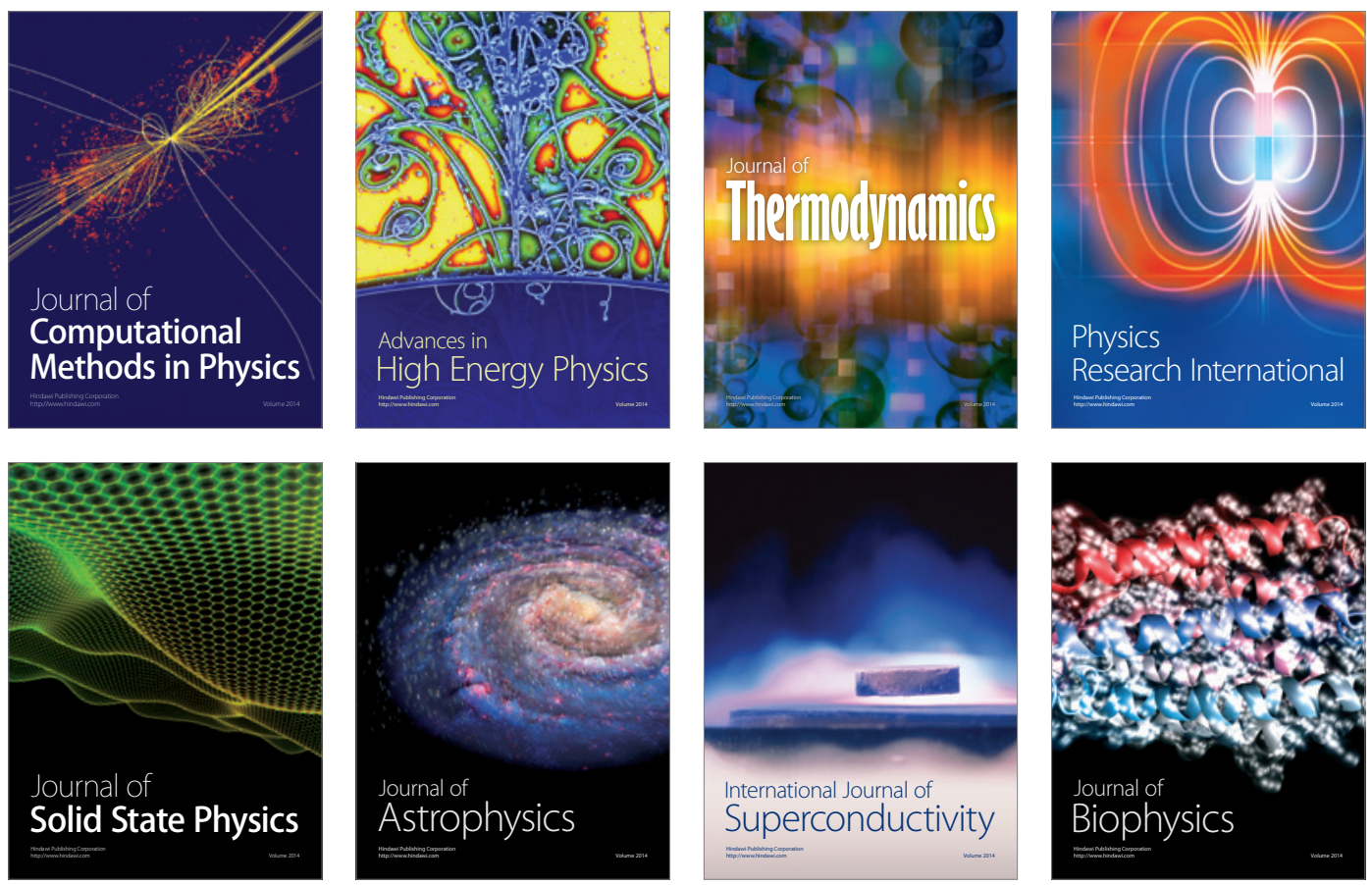
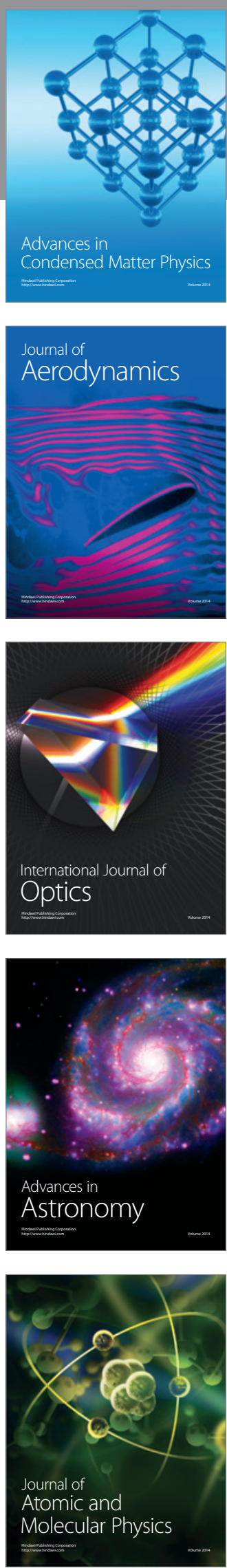